\title{
The Growth of Mixed Populations of Chilomonas paramecium and Tetrahymena pyriformis
}

\author{
By SMILJA MUČIBABIĆ* \\ Zoological Laboratory, University of Cambridge
}

SUMMARY: In mixed cultures where Chilomonas paramecium and Tetrahymena pyriformis $\mathrm{W}$ were in competition for the same dissolved food, the population size of both species (expressed in terms of total number of organisms), as well as the size and shape of individual organisms, were all affected by the presence of the other species. The size of the Chilomonas population was significantly larger in mixed than in single cultures up to the stationary phase, after which the Chilomonas rapidly decreased in numbers and finally died out. The Tetrahymena population was also larger in mixed cultures in the early stages, though by the beginning of the stationary phase this was not so. Only when the Chilomonas had died out did the Tetrahymena population again reach the size of that in single cultures. In terms of total volume of organisms, the growth of the Tetrahymena population was not as good initially as in single cultures. Later, when the Chilomonas population became very small, the total volume of the Tetrahymena population increased and finally reached the size of the population in single cultures.

Chilomonas paramecium and Tetrahymena pyriformis were both more slender in mixed than in single cultures, the differences being statistically significant. Variability of the organisms was greater in mixed cultures and changed with the age of the population. During population growth, the individual size of the Chilomonas and the Tetrahymena changed; but the time-course of change was different for the two species. While the size of the Chilomonas increased in the logarithmic phase and then decreased, the size of the Tetrahymena decreased in the logarithmic phase and increased in the phase of negative growth acceleration. In old cultures, the size of both species was smaller than at the beginning of the stationary phase.

The growth of mixed populations of protozoa, competing for the same food supply, has hitherto been investigated only in organisms feeding on bacteria. In the studies of Gause (1932, 1934, $1935 a, b)$, attention was concentrated on the growth of the protozoa only, while the growth of the bacteria and their influence on the outcome of competition were neglected. The starting-point of the present investigation was a search for two species of protozoa which did not prey on each other and which grew well in the same organic medium, in bacteria-free cultures, and could therefore be used to examine the interaction of competitors not related as predator to prey. Two such species are Chilomonas paramecium Ehrenberg and Tetrahymena pyriformis (Ehrenberg) Lwoff, strain W. Hall (1950) reported that $C$. paramecium was taken as food by $T$. geleii $\mathbf{W}$. In the present experiments, however, neither direct observation nor the results of staining with Lugol's iodine (as a test for starch from ingested Chilomonas) ever suggested that strain $\mathrm{W}$ takes the present Chilomonas as food.

* Present address: Philosophical Faculty, Sarajevo, Yugoslavia. 


\section{METHODS}

The data concerning the strain of Chilomonas paramecium used were given in a previous paper (Mučibabić, 1956). Corliss (1952, 1953a) described the history, systematics and morphology of Tetrahymena pyriformis; the strain W was isolated by C. L. Claff in 1939 (Corliss, 1952), and subcultures for the present investigation were obtained from the Culture Collection of Algae and Protozoa, Botany School, Cambridge. Fresh clones were established to ensure that the populations of the species used in these experiments were homogeneous. It was found that $T$. pyriformis $\mathrm{W}$ and $C$. paramecium both grew well in a solution of $0.1 \%$ Proteose peptone (Difco) and $0.1 \%$ sodium acetate; indeed the Chilomonas grew better in this than in the more usual $0.1 \%$ beef extract (Difco) medium.

The $\mathbf{0 . 5} \mathrm{ml}$. cultures were inoculated with ten organisms of each species, and single populations of each species served as controls, the inoculum of the latter being ten individuals of the Chilomonas or of the Tetrahymena. All cultures were maintained at $22 \cdot 5^{\circ}$. It was necessary to increase the number of observations over the initial period of growth, because the duration of the logarithmic phase varies in individual cultures. Cultures of the Chilomonas, for example, reached the maximum stationary phase in 5-7 days; and cultures of the Tetrahymena in 8-10 days.

Before starting to count, a loopful of every culture was spread over a slope of Bacto-nutrient agar (Difco), subsequently incubated, and examined for signs of bacterial contamination. It is worth mentioning that Tetrahymena pyriformis $\mathrm{W}$ grows on Bacto-nutrient agar, forming small round patches that can easily be mistaken for colonies of bacteria. The cultures were counted completely, or by sampling, and then discarded (Mučibabić, 1956).

\section{Growth of population of Chilomonas paramecium and Tetrahymena pyriformis in terms of total numbers of organisms}

Table 1 shows the growth of mixed and single populations of the Chilomonas and the Tetrahymena in terms of total numbers of organisms. The maximum size of the population of the Chilomonas in mixed cultures was about $170 \%$ of the maximum population in single cultures, and growth was generally better in the mixed populations during the first 11 days. The larger size of population is statistically significant from the sixth day onwards. This large number of Chilomonas in mixed populations was not maintained, however; it began to decrease, slowly at first, and then rapidly, until the end of the twentysecond day. From the twelfth day the number was significantly smaller than in single cultures. Later, there was a slight increase in numbers, but after some fluctuations, the Chilomonas disappeared completely by about the thirty-sixth day.

The course of growth of the population of the Tetrahymena in mixed cultures differs from that of the Chilomonas. During the first 7 days its numbers were larger than in the control single cultures, and this difference was statistically significant after the third day. Later, the numbers were smaller, and from the 
ninth day onwards, significantly so. At the beginning of the maximum stationary phase, the number was about $16 \%$ smaller than in control single cultures. The number did not change significantly until the sudden drop in

Table 1. Growth of single and mixed populations of Chilomonas paramecium and Tetrahymena pyriformis in terms of total number of organisms

$\bar{x}=$ mean value; $s=$ standard deviation; $n=$ number of observations.

Single populations

\begin{tabular}{|c|c|c|c|c|c|}
\hline \multirow{2}{*}{$\begin{array}{c}\text { Age } \\
\text { (days) }\end{array}$} & \multicolumn{3}{|c|}{ C. paramecium } & \multicolumn{2}{|c|}{$T \cdot$ pyriformis } \\
\hline & $\bar{x}$ & $s$ & $n$ & $\bar{x}$ & $s$ \\
\hline 1 & $42 \cdot 4$ & $11 \cdot 2$ & 17 & $210 \cdot 9$ & $55 \cdot 4$ \\
\hline 2 & $308 \cdot 4$ & $71 \cdot 3$ & 19 & 1,046 & 175 \\
\hline $\mathbf{3}$ & $\mathbf{1 , 9 0 5}$ & 1,170 & 20 & 2,200 & 320 \\
\hline 4 & 10,163 & 7,580 & 20 & 4,190 & 1,710 \\
\hline 5 & 47,000 & 25,100 & 20 & 10,640 & 6,510 \\
\hline 6 & 75,300 & 14,000 & 19 & 20,350 & 11,700 \\
\hline 7 & 79,600 & 6,900 & 10 & 27,620 & 7,590 \\
\hline 8 & 79,600 & 7,500 & 7 & 41,600 & 10,800 \\
\hline 9 & 81,400 & 7,500 & 7 & 44,600 & 7,700 \\
\hline 10 & 75,600 & 12,700 & 7 & 42,700 & 7,300 \\
\hline 11 & 78,000 & 6,000 & 7 & 45,600 & 5,500 \\
\hline 12 & 84,250 & 13,700 & 4 & 46,500 & 3,800 \\
\hline 13 & 80,600 & 5,500 & 3 & 45,600 & 3,500 \\
\hline 14 & 92,000 & 13,200 & 4 & 42,250 & 1,200 \\
\hline 15 & 85,300 & 1,200 & 3 & 41,500 & 15,200 \\
\hline 16 & 83,000 & 12,400 & 3 & 42,600 & 2,100 \\
\hline 17 & 79,300 & 8,100 & $\mathbf{3}$ & 46,000 & 3,600 \\
\hline 18 & 84,000 & 6,200 & 3 & 45,300 & 2,500 \\
\hline 19 & 81,600 & 20,300 & 3 & 47,000 & 1,410 \\
\hline 20 & 77,500 & 2,000 & 2 & 49,000 & 5,600 \\
\hline 21 & 73,000 & - & $\mathbf{1}$ & 41,000 & - \\
\hline 22 & 86,000 & 11,300 & 2 & 43,000 & - \\
\hline 23 & 80,000 & - & $\mathbf{1}$ & 38,000 & - \\
\hline 24 & 77,000 & 0 & 2 & 56,000 & - \\
\hline 25 & 69,000 & $\ldots$ & 1 & 二 & - \\
\hline 26 & 73,500 & 2,000 & 2 & - & - \\
\hline 27 & 77,000 & $\ldots$ & 1 & 50,000 & - \\
\hline 28 & 82,500 & 7,700 & 2 & 59,000 & - \\
\hline 29 & 76,000 & - & 1 & 51,000 & - \\
\hline 30 & 73,000 & 9,800 & 2 & 49,000 & - \\
\hline 31 & 71,000 & $\longrightarrow$ & $\mathbf{1}$ & 50,000 & - \\
\hline 32 & 77,500 & 6,300 & 2 & 55,000 & - \\
\hline 33 & 75,000 & - & 1 & 47,000 & - \\
\hline 34 & - & - & - & 42,000 & - \\
\hline 35 & 81,000 & - & 1 & - & - \\
\hline 36 & 73,000 & - & 1 & 50,000 & - \\
\hline
\end{tabular}

Mixed populations

\begin{tabular}{|c|c|c|c|c|c|}
\hline \multicolumn{3}{|c|}{ C. paramecium } & \multicolumn{3}{|c|}{$T \cdot$ pyriformis } \\
\hline $\bar{x}$ & $s$ & $n$ & $\bar{x}$ & $s$ & $n$ \\
\hline $46 \cdot 6$ & $13 \cdot 1$ & 17 & $231 \cdot 2$ & $79 \cdot 6$ & 17 \\
\hline $358 \cdot 8$ & 180 & 18 & 976 & 314 & 13 \\
\hline 1,785 & 631 & 20 & 2,235 & 710 & 20 \\
\hline 11,400 & $7,4,40$ & 18 & 6,720 & 3,500 & 18 \\
\hline 53,600 & 32,900 & 18 & 19,520 & 10,030 & 18 \\
\hline 128,600 & 27,500 & 15 & 31,030 & 6,090 & 15 \\
\hline 135,100 & 26,500 & $\mathbf{9}$ & 33,230 & 4,330 & 9 \\
\hline 126,900 & $\mathbf{3 1 , 3 0 0}$ & 10 & 32,600 & 2,700 & 10 \\
\hline 111,600 & 12,600 & 9 & 34,770 & 6,300 & 9 \\
\hline 96,800 & 31,700 & 9 & 37,200 & 6,100 & 9 \\
\hline 97,200 & 16,400 & 9 & 35,200 & 3,600 & 9 \\
\hline 70,900 & 20,100 & 8 & 33,370 & 4,900 & 8 \\
\hline 36,000 & $\longrightarrow$ & $\mathbf{5}$ & 36,000 & 1,800 & 5 \\
\hline 23,300 & 一 & 9 & 36,100 & 6,500 & 9 \\
\hline 15,400 & 一 & 5 & 39,000 & 5,500 & $\mathbf{5}$ \\
\hline 13,600 & - & $\mathbf{5}$ & 35,200 & 6,000 & $\mathbf{5}$ \\
\hline 14,800 & 一 & $\mathbf{5}$ & 34,600 & 6,700 & $\mathbf{5}$ \\
\hline 4,400 & - & 5 & 37,200 & 9,100 & $\mathbf{5}$ \\
\hline 6,000 & - & 5 & $\mathbf{3 8 , 0 0 0}$ & 5,300 & 5 \\
\hline 5,800 & 一 & 4 & 40,000 & 5,200 & 4 \\
\hline 1,300 & 一 & 3 & 33,600 & 4,900 & 3 \\
\hline 148 & - & 3 & $\mathbf{3 3 , 3 0 0}$ & 5,500 & $\mathbf{3}$ \\
\hline 360 & - & 3 & 36,600 & 3,200 & $\mathbf{3}$ \\
\hline 730 & - & 3 & 43,300 & 13,600 & 3 \\
\hline 830 & - & 3 & 36,300 & 1,500 & $\mathbf{3}$ \\
\hline 1,028 & - & $\mathbf{3}$ & 39,000 & 4,400 & $\mathbf{3}$ \\
\hline 600 & - & 3 & 46,000 & 7,000 & 3 \\
\hline 1,338 & - & $\mathbf{3}$ & 43,700 & 4,400 & 3 \\
\hline 600 & $\ldots$ & 3 & 43,600 & 5,100 & $\mathbf{3}$ \\
\hline 2,190 & - & 1 & 44,000 & - & 1 \\
\hline 230 & - & 3 & 38,600 & 7,000 & 3 \\
\hline 940 & - & 3 & 38,700 & 7,400 & 3 \\
\hline 600 & - & 3 & 47,000 & 6,900 & 3 \\
\hline 0 & - & 3 & 44,000 & 7,100 & $\mathbf{3}$ \\
\hline $\mathbf{3 6 0}$ & - & $\mathbf{3}$ & 43,600 & 2,300 & $\mathbf{3}$ \\
\hline 0 & - & $\mathbf{3}$ & 39,600 & 2,900 & $\mathbf{3}$ \\
\hline
\end{tabular}

the population of the Chilomonas began; it then showed an increase, so that near the end of the experiment the size of population did not differ significantly from that of the Tetrahymena in control cultures.

In the single cultures, when the populations of the Chilomonas and the Tetrahymena reached the maximum stationary phase, their numbers were maintained with but small fluctuations until the end of observations (36 days). 
Growth of population of Chilomonas paramecium and Tetrahymena pyriformis in terms of the total volume of organisms

Previous measurements of the size of Tetrahymena pyriformis, strain W, by Loefer (1952) and Corliss (1953 $a$ ), were made on fixed organisms. Preliminary observations showed, however, that shrinkage occurred during fixation, but was least in Champy's fixative. In order to test whether reliable estimates of the volume in life could be obtained from organisms fixed with this fixative, photographs were taken, first of a living organism T. pyriformis, and then of

Table 2. Growth of single and mixed populations of Chilomonas paramecium and Tetrahymena pyriformis in terms of total volume of organisms

\begin{tabular}{|c|c|c|c|c|}
\hline \multirow{2}{*}{$\begin{array}{c}\text { Age } \\
\text { (days) }\end{array}$} & \multicolumn{2}{|c|}{ Single populations } & \multicolumn{2}{|c|}{ Mixed populations } \\
\hline & C. paramecium & $\begin{array}{c}T \cdot \text { pyriformis } \\
\text { (thousan }\end{array}$ & $\begin{array}{l}\text { C. paramecium } \\
\left.\text { ds of } \mu .^{3}\right)\end{array}$ & T. pyriformis \\
\hline 0 & 12 & 175 & 12 & 175 \\
\hline 2 & 312 & 12,720 & 535 & 10,780 \\
\hline 4 & 19,832 & 92,431 & 22,048 & 103,864 \\
\hline 6 & 95,255 & 474,766 & 231,223 & 369,971 \\
\hline 8 & 101,510 & $1,287,894$ & 133,753 & 433,547 \\
\hline 10 & 131,998 & 728,419 & 113,740 & 565,626 \\
\hline 12 & 135,306 & 575,252 & 54,664 & 323,989 \\
\hline 14 & 127,236 & 450,512 & 20,574 & 327,319 \\
\hline 16 & 92,130 & 434,520 & 14,090 & 312,963 \\
\hline 18 & 81,816 & 487,519 & 4,176 & 289,862 \\
\hline 20 & 86,955 & 455,651 & 8,462 & 372,760 \\
\hline 22 & 84,108 & 488,179 & 157 & 287,012 \\
\hline 24 & 95,480 & 536,928 & 958 & 339,082 \\
\hline 26 & 63,798 & 478,300 & 1,245 & 380,367 \\
\hline 28 & 68,145 & 436,442 & 1,545 & 464,444 \\
\hline 30 & 71,686 & 427,672 & 2,146 & 448,174 \\
\hline 32 & 76,570 & 551,760 & 905 & 446,056 \\
\hline 34 & 95,706 & 410,550 & 0 & 356,136 \\
\hline
\end{tabular}

the same organism after fixation in Champy's fluid and washing in Da Fano's solution (Corliss, 1953b). Sixteen organisms were photographed in this way (Pl. 1, fig. 1), and the size of the live and fixed organisms (length and greatest width) was measured from the photographs. From the series of differences, $t$ was determined (Fisher, 1950; see 'Significance of mean of a small sample'). Its value (2.59) both for length and width (for 15 degrees of freedom) showed that the differences in size between living organisms and organisms fixed in Champy's fixative were statistically significant ( $P$ lies between 0.05 and 0.02 ). It was evident that only measurements of living organisms were of value for a quantitative study of changes in size. Accordingly, photographs of living organisms in single and mixed cultures were taken every second day throughout the growth of the population (Pl. 1, figs. 2-4). The length and maximal width of the organism in sharp focus were measured, and the average volume of individual organisms in single and in mixed populations calculated.

These data were used for the calculation of the total volume of populations 
in single and mixed cultures. The total numbers of organisms (Table 1) were multiplied by the corresponding value for the volume of individual organisms at the same age (Tables 3 and 4 ); the results are shown in Table 2. They indicate first, that the maximum size of population (total volume or 'biomass') reached in single cultures was not maintained for as long a time as when expressed as total number of organisms (Table 1).

The growth of the Chilomonas populations in mixed cultures, expressed as total volume of organisms, followed the same course in time as when expressed as total number of organisms; but the growth of the Tetrahymena in mixed cultures in terms of total volume was different from that in terms of total number; growth was worse than in the control cultures (except for 1 day), the maximum yield being only $44 \%$ of that in the single population.

It is to be noted that the total volume of the Tetrahymena, either in single or mixed populations, was always greater than the total volume of the population of the Chilomonas. The maximum size of the single population of the Chilomonas was about $231 \times 10^{6} \mu .^{3}$, while that of the Tetrahymena was about $1288 \times 10^{6} \mu .^{3}$. These differences occurred in cultures with the same kind and quantity of nutrient medium. They imply that the degree of utilization of the nutrient medium depended on physiological characteristics of the species.

Changes in size and shape of Chilomonas paramecium and of Tetrahymena pyriformis during the growth of single and mixed populations

Values for length and width, for the ratio of length to width, as well as for the volume, of individual organisms of the Chilomonas and the Tetrahymena, during the growth of single and mixed populations, are shown in Tables 3 and 4 . These list the mean value $(\vec{x})$ and coefficient of variation (c.v.)* of the length and width, and of their ratio; the average volume $(V)$, and the number of observations $(n)$. The ratio of length to width was calculated for each organism measured. The average volume of the Tetrahymena was calculated in the same way as the volume of the Chilomonas, that is, $V=\frac{4}{3} \frac{L}{2}\left(\frac{W}{2}\right)^{2}$ because it appeared that the form of both organisms approximates most nearly to an ellipsoid.

Tables 3 and 4 show that the size and shape of both organisms depended on the age of the population and on the presence or absence of the other species (see also Pl. 1, figs. 2-4). It is clear that the Tetrahymena and the Chilomonas differed not only (and obviously) in average size and shape, but also in the sequence of changes in size and shape during population growth. The Chilomonas became larger after inoculation, while the Tetrahymena became smaller. The increase in size of the Chilomonas, both in single and mixed cultures, was confined to the early stages of growth of the population. During this period, and in the logarithmic phase of growth, the size of the Tetrahymena in single culture decreased (Pl. 1, fig. 2a); subsequently, in the phase

* Coefficient of variation = relative standard deviation, that is, the standard deviation expressed as a percentage of the mean. 
Table 3. Changes in size and shape of Chilomonas paramecium during population growth in single and mixed cultures

$L=$ length $(\mu.) ; W=$ width $(\mu.) ; V=$ volume $\left(\mu .^{3}\right) ; \mathbf{c . v} .=$ coefficient of variation

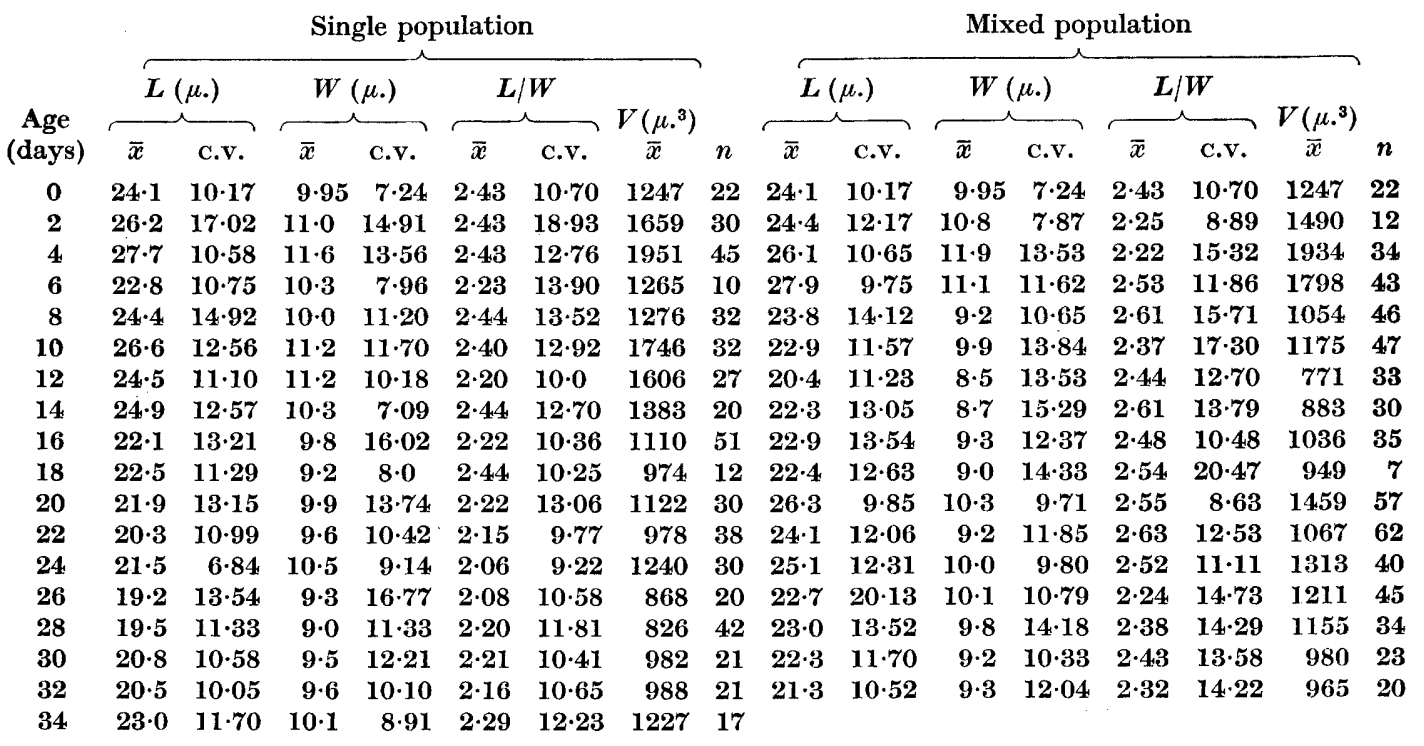

Table 4. Changes in size and shape of Tetrahymena pyriformis-W during population growth in single and mixed cultures

$\boldsymbol{L}=$ length $(\mu.) ; \boldsymbol{W}=$ width $(\mu.) ; V=$ volume $\left(\mu .^{3}\right) ; \mathrm{c.v} .=$ coefficient of variation

Single population

\begin{tabular}{|c|c|c|c|c|c|c|c|c|c|c|c|c|c|c|c|c|}
\hline \multirow{2}{*}{$\begin{array}{l}\text { Age } \\
\text { (days) }\end{array}$} & \multicolumn{2}{|c|}{$L(\mu)}$. & \multicolumn{2}{|c|}{$\boldsymbol{W}\left(\mu_{\bullet}\right)$} & \multicolumn{2}{|c|}{$\boldsymbol{L} / \boldsymbol{W}$} & \multirow{2}{*}{$\begin{array}{c}V\left(\mu .^{3}\right) \\
\bar{x}\end{array}$} & \multirow[b]{2}{*}{$n$} & \multicolumn{2}{|c|}{$L(\mu)}$. & \multicolumn{2}{|c|}{$\boldsymbol{W}\left(\mu_{\cdot}\right)$} & \multicolumn{2}{|c|}{$\boldsymbol{L} / \boldsymbol{W}$} & \multirow{2}{*}{$\begin{array}{c}V\left(\mu .^{\mathbf{3}}\right) \\
\vec{x}\end{array}$} & \multirow[b]{2}{*}{$n$} \\
\hline & $\overline{\boldsymbol{x}}$ & c.v. & $\bar{x}$ & c.v. & $\bar{x}$ & c.v. & & & $\bar{x}$ & c.v. & $\bar{x}$ & c.v. & $\bar{x}$ & c.v. & & \\
\hline 0 & $69 \cdot 1$ & $11 \cdot 1$ & $22 \cdot 0$ & $12 \cdot 77$ & $3 \cdot 17$ & $11 \cdot 36$ & & 16 & $69 \cdot 1$ & $11 \cdot 14$ & $22 \cdot 0$ & $12 \cdot 77$ & $3 \cdot 17$ & $11 \cdot 36$ & 17,491 & 16 \\
\hline 2 & $42 \cdot 8$ & & $3 \cdot 3$ & $11 \cdot 8$ & $1 \cdot 85$ & & & 22 & $43 \cdot 6$ & & $22 \cdot 0$ & & & & & \\
\hline 4 & $49 \cdot 3$ & & $29 \cdot 2$ & & 1.69 & & & 36 & $48 \cdot 8$ & & $24 \cdot 6$ & 46 & 1.98 & $7 \cdot 58$ & & \\
\hline 6 & $56 \cdot 9$ & $12 \cdot 39$ & $28 \cdot 0$ & 18 & $2 \cdot 06$ & 11. & & 15 & 50 & & $21 \cdot 3$ & & 2.35 & & & 12 \\
\hline 8 & $62 \cdot 4$ & & $30 \cdot 8$ & & $2 \cdot 04$ & & & 39 & 54 & & $21 \cdot 6$ & & & & & \\
\hline 10 & $54 \cdot 3$ & & 24.5 & & $2 \cdot 25$ & & & 23 & 55 & & $22 \cdot 9$ & $22 \cdot 49$ & $2 \cdot 36$ & & & 36 \\
\hline 12 & $52 \cdot 1$ & & $21 \cdot 3$ & & $2 \cdot 46$ & & & 30 & $47 \cdot 8$ & & $19 \cdot 7$ & $8 \cdot 02$ & $2 \cdot 43$ & & & 58 \\
\hline 14 & $46 \cdot 2$ & & $21 \cdot 0$ & & $2 \cdot 20$ & $7 \cdot 27$ & & 24 & $49 \cdot 1$ & & $18 \cdot 7$ & 70 & & & & 32 \\
\hline 16 & $41 \cdot 8$ & 11 & $21 \cdot 6$ & & 1.94 & & & 80 & 42 & & $20 \cdot 0$ & .55 & $2 \cdot 14$ & & & 40 \\
\hline 18 & $43 \cdot 3$ & & $21 \cdot 8$ & & 1.93 & 18 . & & 44 & $40 \cdot 0$ & & $19 \cdot 3$ & $2 \cdot 12$ & $2 \cdot 07$ & & & 49 \\
\hline 20 & $41 \cdot 8$ & & $20 \cdot 9$ & & $2 \cdot 01$ & & & 33 & $42 \cdot 4$ & & $20 \cdot 5$ & $5 \cdot 17$ & $2 \cdot 09$ & & & 51 \\
\hline 22 & $48 \cdot 3$ & $13 \cdot 60$ & $21 \cdot 2$ & $14 \cdot 91$ & $2 \cdot 29$ & $11 \cdot 35$ & & 16 & $42 \cdot 9$ & & $19 \cdot 6$ & $15 \cdot 61$ & $2 \cdot 20$ & & & 40 \\
\hline 24 & $43 \cdot 2$ & & $20 \cdot 6$ & $9 \cdot 76$ & $2 \cdot 10$ & 8.57 & & 43 & $40 \cdot 2$ & & $19 \cdot 3$ & $9 \cdot 50$ & $2 \cdot 10$ & & & 56 \\
\hline 26 & $43 \cdot 1$ & & $20 \cdot 6$ & & $2 \cdot 10$ & & & 41 & $43 \cdot 1$ & & $20 \cdot 8$ & $21 \cdot 44$ & $2 \cdot 10$ & & & 41 \\
\hline 28 & $46 \cdot 6$ & & $19 \cdot 7$ & $9 \cdot 24$ & $2 \cdot 37$ & & & 61 & $49 \cdot 8$ & 12.93 & $20 \cdot 2$ & $21 \cdot 34$ & $2 \cdot 54$ & $18 \cdot 78$ & & \\
\hline 30 & $40 \cdot 9$ & & $20 \cdot 2$ & $11 \cdot 24$ & $2 \cdot 04$ & $8 \cdot 86$ & & 23 & $50 \cdot 7$ & & $19 \cdot 6$ & $29 \cdot 80$ & $2 \cdot 70$ & $18 \cdot 89$ & 10,186 & 24 \\
\hline 32 & $45 \cdot 2$ & $10 \cdot 82$ & $20 \cdot 6$ & & $2 \cdot 19$ & & 10,032 & 39 & $49 \cdot 5$ & $19 \cdot 4$ & $21 \cdot 1$ & $32 \cdot 89$ & $2 \cdot 4,4$ & $16 \cdot 39$ & 11,526 & 53 \\
\hline 34 & $43 \cdot 2$ & 11.94 & $20 \cdot 8$ & $6 \cdot 30$ & $2 \cdot 07$ & $9 \cdot 18$ & 9,775 & 25 & $40 \cdot 7$ & $9 \cdot 43$ & $19 \cdot 5$ & $19 \cdot 60$ & $2 \cdot 01$ & $7 \cdot 46$ & 8,094 & 61 \\
\hline
\end{tabular}


of negative growth acceleration, it became larger again (P1. 1, fig. 2b). This increase in size was followed by a sudden decrease, at the beginning of the stationary phase (Pl. 1, fig. 2c). In the mixed population, the Tetrahymena never reaches the maximum size of organisms in the single population ( $\mathrm{Pl}$. 1, fig. $4 a-d)$; and later its decrease in size was greater than in control cultures. Nevertheless, the size of the Tetrahymena in mixed populations increased again when the Chilomonas was nearly suppressed (Pl. 1, fig. 4d).

The decrease in size of the Chilomonas in the phase of negative growth acceleration was greater in single than in mixed cultures. When the single population of the Chilomonas stopped growing, however, the size of individuals increased again ( $\mathrm{Pl}$. 1, fig. 3c), surpassing the size of organisms in mixed cultures, but did not become as large as that of organisms in the early stages of growth of the single culture. The individual size of the Chilomonas in mixed cultures also began to increase as soon as the density of the population became low, and surpassed that of organisms in control single cultures of approximately the same age.

The ratio of length to width has been adopted as a quantitative expression of the shape of the organisms. Generally speaking, the values of this ratio are larger in mixed populations than in single. This means that the organisms are more slender when grown in mixed cultures than in single cultures; and these differences in shape are more accentuated in the Tetrahymena than in the Chilomonas.

Tetrahymena pyriformis $\mathrm{W}$ was plump in the logarithmic phase of growth; but in the phase of negative growth acceleration, and in the first part of the stationary phase, the organisms were slender. In older cultures, both slender and plump organisms were present. Chilomonas paramecium, however, was plump only in single cultures more than 20 days old. In mixed cultures it was more slender, except in the logarithmic phase of growth when the organisms were plumper than in single cultures.

Tetrahymena pyriformis $\mathrm{W}$ swam more rapidly in the mixed cultures than in single culture. This greater speed of swimming was not observed at the beginning of population growth, but was very noticeable from the phase of negative growth acceleration onwards. In old cultures, however, it was not observed. From their stream-lined shape in cultures 8 days old (Pl. 1, fig. 2b) one might indeed expect that organisms from the mixed populations would be faster swimmers than those grown in single culture.

Tables 3 and 4 show that, on the whole, the coefficients of variation of length, width and of their ratio, are larger in mixed populations. It is interesting that variation in the shape of the Chilomonas was more accentuated than variation in size; while for the Tetrahymena the converse was true.

Variation of the Chilomonas in single population was greater only at the beginning of the maximum stationary phase; while in a mixed population high values of the coefficients appeared almost throughout population growth.

Increased variation of the Tetrahymena in single cultures was exhibited at the end of the phase of negative growth acceleration, and at the beginning and in the middle of the maximum stationary phase. In mixed cultures, 
however, the first increase in the coefficients of variation occurs at the beginning of the maximum stationary phase. The values of the coefficients of variation for the Tetrahymena were maximal when the Chilomonas was almost completely suppressed, and the population of the Tetrahymena had begun to increase. How great is the variation in size and shape of the Tetrahymena in mixed cultures at this time can be seen from Pl. 1, fig. $4 d$.

The values of length, width, and the ratio of length to width of the Chilomonas and the Tetrahymena, in single and mixed cultures, were compared statistically, using the $t$-test of significance. The results showed that differences of means of length and width of organisms in single and mixed populations were significant only from the fourth to the sixth day after inoculation. On the other hand, the difference in shape of the Tetrahymena from single and mixed cultures is significant from the beginning of measurements; it was almost significant for the Chilomonas also. As might be expected, the significance of means changes during population growth; but on the whole the differences between the organisms from single and mixed cultures are significant.

The maximal and minimal values of length, width and their ratio in single and mixed populations were also statistically compared. They were all highly significant $(P<0 \cdot 001)$.

\section{DISCUSSION}

These experiments have shown that the growth of two protozoan species in mixed populations is not as simple a matter as certain mathematical theories have suggested. Statistically significant differences in the population size of Chilomonas paramecium and Tetrahymena pyriformis $\mathrm{W}$, between single and mixed cultures, appeared at the beginning of population growth, and implied better growth of both species in mixed populations. Later, however, there was significantly better growth of both species in single populations. Now C. paramecium, a species of which the maximum population was nearly twice as large in mixed as in single cultures, was suppressed by $T$. pyriformis $\mathbf{W}$, of which the maximal population was larger in single than in mixed cultures. According to theories of competition between two species, however, one would not expect suppression of the species that grows better in mixed cultures from the logarithmic to the stationary phase.

The reason for the better initial growth of both species in mixed cultures is unknown. Obvious possibilities are: that certain products of the metabolism of one species favoured population growth of the other; or that one species removed something from the medium and so made it more suitable for the growth of the other species. Whatever its nature, this conditioning of the medium took a few days, since significantly greater numbers of the Tetrahymena in mixed cultures only appeared on the fourth day, and those of the Chilomonas only on the sixth day after inoculation. The greater concentration of carbon dioxide in mixed cultures (due to a larger number of organisms) might conceivably stimulate growth of the Chilomonas population (Jahn, 1936; Pace \& Ireland, 1945). 
The growth rate (Mučibabić, 1956) of the population of the Tetrahymena, both in single and mixed cultures, was greater than that of the Chilomonas population during the first day; but later, the growth rate of the Chilomonas was constantly greater. From its growth rate, as well as from the maximum population size, one might expect the Chilomonas to win in competition with the Tetrahymena; but the Chilomonas was suppressed.

On general grounds, Huxley (1942) emphasized that change in environmental conditions may affect the results of competition, and Hutchinson (1941) showed how a successional series in any system may be caused by internal or external changes modifying properties which bear directly on the struggle for existence. Crombie (1945) also claimed that the mathematical theories of competition between two species hold only so long as the conditions of experiment remain constant; and it seems evident that in cultures such as those examined here, there must be a steady change of environmental conditions throughout the life of the culture.

Salt (1932) showed for a very different organism, namely the dipteran Lucilia sericata, that the population growth assumed a different appearance according to whether population size was expressed as number of organisms or as weight of organisms. This result is analogous to the observed difference in the effects of competition between the Chilomonas and the Tetrahymena used here, when the size of the population is expressed as numbers or volume of organisms.

McVeigh \& Brown (1954) compared the growth of the flagellates Chlamydomonas chlamydogama and Haematococcus pluvialis, in single and mixed population, when the cultures were 2 and 3 weeks old. They found better growth in single cultures after 2 weeks, and in mixed cultures after 3 weeks. Since they did not follow the growth of the population throughout, their results are not fully comparable with the findings of the present experiments, though to some extent they confirm the observed better growth of the Chilomonas and the Tetrahymena in a mixed population at a particular age of the culture.

The observation that the size of the Tetrahymena and Chilomonas organisms in mixed culture was significantly different from the size of organisms in single culture, and that there were marked differences between the sizes of organisms of the same culture at different ages of the population, implies that one cannot with safety measure a certain number of organisms from a single culture and use the mean values obtained from such measurements for calculating the size of population, in terms of total volume of organisms, for cultures of different age and composition, as did Gause $(1935 a, b)$ in his experiments on competition between two species of ciliates.

The data on variability suggest that the conditions of competition between two species induce an increased variability in size and shape of organisms in mixed cultures. This was particularly striking in the Tetrahymena at the time when the Chilomonas was nearly suppressed. It implies that changes occurred both in volume and in surface area of the organisms; in the Tetrahymena, either the kineties increased in length, or the distance between them increased during an increase in surface area, or both may have happened simultaneously. These are matters for future investigation. 
Ormsbee (1942) noted that there was variability in size of Tetrahymena pyriformis $\mathrm{W}$ in the stationary phase of population growth; but he judged changes in size of organisms from changes in length only, and claimed that the ratio of length to width did not vary significantly during population growth. The present experiments have shown, however, that profound changes in the ratio of length to width occurred in the logarithmic phase of population growth of $T$. pyriformis W. Ormsbee also observed (as here described, p. 565) a decrease in size of the Tetrahymena during the logarithmic phase of growth and a subsequent increase in size in the stationary phase; in the present experiments, however, an increase in size was observed in the phase of negative growth acceleration. In contradiction to my results and to those of Ormsbee, however, Slater \& Elliott (1951) found that the volume of individual organisms of $T$. geleii was maximal at the end of the logarithmic phase. It was claimed by Loefer (1952) that all strains of $T$. geleii reached full size after $48 \mathrm{hr}$.; in my experiments, however, full size was not reached until 8 days after inoculation. Loefer, however, used a medium differing from mine in kind and quantity, and maintained his cultures at a higher temperature.

The observed decrease in size of organisms in old cultures, though the total numbers remained constant, may be connected with progressive deficiency in the food available, for Weis (1954) also observed decrease in size in Tetrahymena pyriformis in starving cultures. Harding (1937) measured the size of Glaucoma pyriformis (Tetrahymena pyriformis) in cultures without food, and also found that size decreased rapidly at the beginning of starvation, while the organisms were still multiplying.

I wish to express my gratitude to the heads of the Botany School and of the Department of Zoology, Cambridge University, for research facilities, and to the many assistants who have spared no trouble in helping me. My sincere thanks are due to Professor E. G. Pringsheim, Dr G. Salt, Professor S. Stanković, Professor G. E. Briggs and Mr E. A. George, for stimulating discussions and useful suggestions in relation to my work. In particular I am indebted to Dr L. E. R. Picken for constant help and encouragement.

\section{REFERENCES}

Conliss, J. O. (1952). Comparative studies on holotrichous ciliates in the ColpidiumGlaucoma-Leucophrys-Tetrahymena Group I. General consideration and history of strains in pure culture. Trans. Amer. micr. Soc. 71, 159.

Conliss, J. O. (1953a). Comparative studies on holotrichous ciliates in the Colpidium-Glaucoma-Leucophrys-Tetrahymena Group II. Morphology, life cycles and systematic status of strains in pure culture. Parasitology, 43, 49.

Corliss, J. O. $(\mathbf{1 9 5 3} \mathrm{b})$. Silver impregnation of ciliated protozoa by the ChattonLwoff technique. Stain Tech. 28, 97.

Crombie, A. C. (1945). On competition between different species of graminivorous insects. Proc. Roy. Soc. B, 132, 362.

Fisher, R. A. (1950). Statistical Methods for Research Workers, 11th ed. Edinburgh: Oliver and Boyd.

Gause, G. F. (1932). Experimental studies on the struggle for existence. 1. Mixed population of two species of yeast. J. exp. Biol. 9, 389 .

Gause, G. F. (1934). The Struggle for Existence, p. 163. Baltimore: Williams and Wilkins Co. 
Journal of General Microbiology, Vol. 16, No. 3

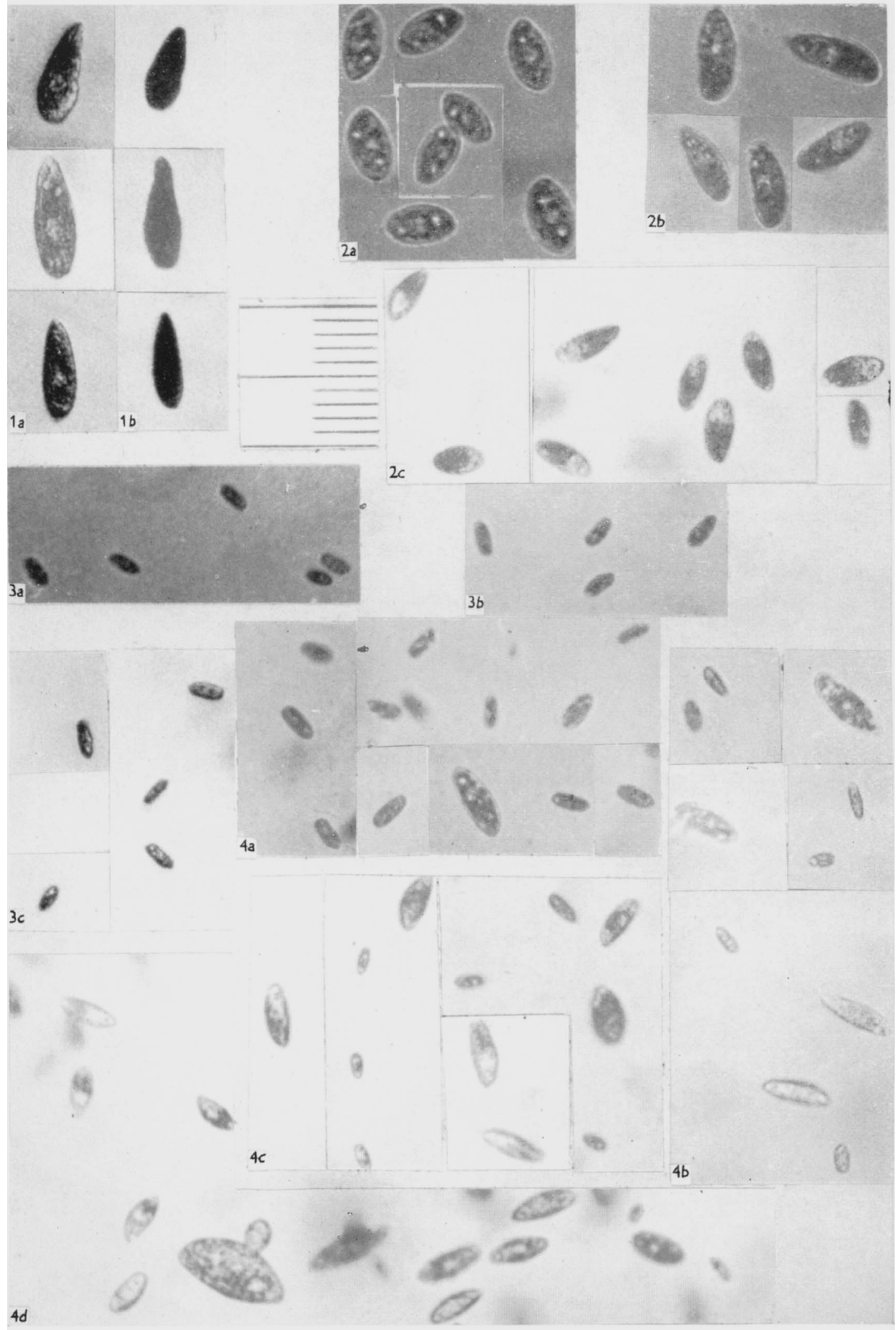

S. Mučibabić-Mixied popelation growtir of protozon. Platte 1 
Gause, G. F. (1935a). Vérifications expérimentales de la théorie mathématique de la lutte pour la vie. Actualités sci. industr. 277, ix, 62.

GaUse, G. F. $(1935 b)$. Experimentelle Untersuchungen über die Konkurrenz zwischen Paramecium caudatum und Paramecium aurelia. Arch. Protistenk. 84, 207.

Hall, R. P. (1950). Phytoflagellates as a source of food for Tetrahymena. Proc. Amer. Soc. Protozool. $1,5$.

Handing, J. P. (1937). Quantitative studies on the ciliate Glaucoma. II. The effects of starvation. J. exp. Biol. 14, 431.

Hutchinson, G. E. (1941). Ecological aspects of succession in natural populations. Amer. Nat. 75, 406.

Huxley, J. S. (1942). Evolution: The Modern Synthesis, pp. 119-120. London: Allen and Unwin.

$\mathrm{J}_{\mathrm{AHN}}$, T. L. (1936). Effect of aeration and lack of $\mathrm{CO}_{2}$ on growth of bacteria-free cultures of Protozoa. Proc. Soc. exp. Biol., N.Y. 33, 494.

Loefer, J. B. (1952). Some observations on the size of Tetrahymena. J. Morph.90, 407.

MCVeIGH, I. \& Brown, W. H. (1954). In vitro growth of Chlamydomonas chlamydogama Bold and Haematococcus pluvialis Flotow em. Wille in mixed cultures. Bull. Torrey. bot. $\mathrm{Cl} . \mathbf{8 1}, 218$.

Mư̌rrabić, S. (1956). Some aspects of the growth of single and mixed populations of flagellates and ciliates. Effect of different temperatures on the population growth of Chilomonas paramecium. J. exp. Biol. 33, 627.

Ormsbee, R. A. (1942). The normal growth and respiration of Tetrahymena geleii. Biol. Bull., Woods Hole, 82, 423.

Pace, D. M. \& Ireiand, R. L. (1945). The effects of oxygen, carbon dioxide and pressure on growth in Chilomonas paramecium and Tetrahymena geleii Furgason. J. gen. Physiol. 28, 547.

Salt, G. (1932). The natural control of the sheep blow fly, Lucilia sericata Meigen. Bull. ent. Res. 23, 235.

Slater, J. V. \& EluiotT, A. M. (1951). Volume change in Tetrahymena in relation to age of the culture. Proc. Amer. Soc. Protozool. 2, 20.

WEIS, D. (1954). Observations on size reversibility in cultures of Tetrahymena pyriformis (abstr.). J. Protozool. 1 (Suppl.), 10.

\section{EXPLANATION OF PLATE}

Chilomonas paramecium and Tetrahymena pyriformis $\mathrm{W}$ photographed alive (except for Fig. $1 b$ ) without a coverslip in single and mixed cultures. The smaller divisions on the scale are $10 \mu$.

Fig. 1. T. pyriformis: $(a)$ three living individuals; $(b)$ the same three individuals fixed in Champy's fluid.

Fig. 2. T. pyriformis in single bacteria-free culture. (a) 4 days old; (b) 8 days old; $(c) 14$ days old.

Fig. 3. C. paramecium in single bacteria-free culture. $(a) 6$ days old; $(b) 8$ days old; $(c) 14$ days old.

Fig. 4. Mixed bacteria-free cultures of C. paramecium and T. pyriformis. (a) 6 days old; (b) 8 days old; (c) 14 days old; $(d) 32$ days old.

(Received 2 October 1956) 